Groups Geom. Dyn. 4 (2010), 693-707

DOI $10.4171 / \mathrm{GGD} / 101$
Groups, Geometry, and Dynamics

(C) European Mathematical Society

\title{
Cofinitely Hopfian groups, open mappings and knot complements*
}

\author{
Martin R. Bridson, Daniel Groves, Jonathan A. Hillman and Gaven J. Martin
}

\begin{abstract}
A group $\Gamma$ is defined to be cofinitely Hopfian if every homomorphism $\Gamma \rightarrow \Gamma$ whose image is of finite index is an automorphism. Geometrically significant groups enjoying this property include certain relatively hyperbolic groups and many lattices. A knot group is cofinitely Hopfian if and only if the knot is not a torus knot. A free-by-cyclic group is cofinitely Hopfian if and only if it has trivial centre. Applications to the theory of open mappings between manifolds are presented.
\end{abstract}

Mathematics Subject Classification (2010). 20F65, 57M25 (20F67, 20E26).

Keywords. Cofinitely Hopfian, open mappings, relatively hyperbolic, free-by-cyclic, knot groups.

\section{Introduction}

A group $\Gamma$ is said to be Hopfian (or to have the Hopf property) if every surjective endomorphism of $\Gamma$ is an automorphism. It is said to be (finitely) co-Hopfian if every injective endomorphism (with image of finite index) is an automorphism. These properties came to prominence in Hopf's work on self-maps of surfaces [23]. Related notions have since played a significant role in many contexts, for instance combinatorial group theory, e.g. [3], [36], and the study of approximate fibrations [11]. Here we will be concerned with a Hopf-type property that played a central role in the work of Bridson, Hinkkanen and Martin on open and quasi-regular mappings of compact negatively curved manifolds [7].

We say that a group $\Gamma$ is cofinitely Hopfian (or has the cofinite Hopf property) if every homomorphism $\Gamma \rightarrow \Gamma$ whose image is of finite index is an automorphism. This condition is stronger than both the Hopf property and the finite co-Hopf property.

\footnotetext{
*This paper is based upon work supported by the National Science Foundation (USA) and the Marsden Fund (NZ). Bridson was supported by a Senior Fellowship from the EPSRC (UK) and a Royal Society Wolfson Research Merit Award. Hillman was the Grey College Mathematics Fellow for Michaelmas Term of 2008 at Durham University.
} 
In Section 5 we discuss its relationship to other Hopf-type properties. In Section 2 we recall its relationship to open mappings of manifolds.

If a torsion-free group is hyperbolic in the sense of Gromov, then it is cofinitely Hopfian: this can be deduced from Zlil Sela's profound study of the endomorphisms of such groups [36], [35]; see [7]. In the present article, we shall extend this result to cover several other classes of groups associated with non-positive curvature in group theory. Classical rigidity results imply that lattices in semisimple Lie groups with trivial centre and no compact factors are cofinitely Hopfian (Section 4). In the other classes that we consider the issue is more subtle. These classes are the toral relatively hyperbolic groups, free-by-cyclic groups, and (classical) knot groups. The following are special cases of the results that we prove in each case.

Theorem A. If a toral relatively hyperbolic group is co-Hopfian then it is cofinitely Hopfian.

Theorem B. Let $F$ be a finitely generated free group and let $\Gamma=F \rtimes_{\theta} \mathbb{Z}$. The following conditions are equivalent:

(1) $\Gamma$ is cofinitely Hopfian;

(2) $\Gamma$ has trivial centre;

(3) $\theta$ has infinite order in $\operatorname{Out}(F)$.

Theorem C. Let $K$ be a knot in $\mathbb{S}^{3}$. The fundamental group of $\mathbb{S}^{3} \backslash K$ is cofinitely Hopfian if and only if $K$ is not a torus knot.

If one can prove that the groups in a certain class are cofinitely Hopfian, one can immediately deduce constraints on the nature of open mappings of manifolds whose fundamental groups lies in that class (see Section 2). To exemplify this, in Section 7 we use Theorem $\mathrm{C}$ to prove:

Theorem D. Let $K \subset \mathbb{S}^{3}$ be a knot. If $K$ is not a torus knot, then every proper open self-mapping of $\mathbb{S}^{3} \backslash K$ is homotopic to a homeomorphism.

\section{Topological motivation}

The work of John Walsh [45], Theorem 4.1, and Steven Smale [40] establishes a strong connection between the cofinite Hopf property of groups and open mappings of quite general spaces. For simplicity we state their results only for manifolds, which is sufficient for our purposes. Recall that a map is proper if the preimage of each compact set is compact, and a light mapping is one for which the preimage of every point is a totally disconnected space, for instance a Cantor set. 
Proposition 2.1. Let $M_{1}$ and $M_{2}$ be connected manifolds (possibly with boundary). If a map $f: M_{1} \rightarrow M_{2}$ is proper, open and surjective, then the index of $f_{*} \pi_{1} M_{1}$ in $\pi_{1} M_{2}$ is finite. Moreover, $f$ induces a surjection on rational homology.

In the opposite direction we have:

Theorem 2.2. If $M$ and $N$ are compact connected PL manifolds and $f: M \rightarrow N$ is a map with $\left[\pi_{1} N: f_{*} \pi_{1} M\right]<\infty$, then $f$ is homotopic to a light open mapping.

In [7] these results were used in tandem with the fact that torsion-free hyperbolic groups are cofinitely Hopfian to study discrete open mappings between manifolds of negative curvature. Motivation for this study comes from the Lichnerowicz problem of identifying those $n$-manifolds which admit rational endomorphisms [29], and from a desire to extend the scope of Mostow rigidity.

\section{Toral relatively hyperbolic groups}

A finitely generated group $\Gamma$ is toral relatively hyperbolic if it is torsion-free and hyperbolic relative to a collection of free abelian subgroups. We refer the reader to [10] for a more expansive definition. For our purposes, the following observations will be more useful than the technicalities of the definition.

The following families of groups are toral relatively hyperbolic:

- torsion-free (word) hyperbolic groups;

- fundamental groups of finite-volume or geometrically finite hyperbolic manifolds whose cusp cross-sections are tori;

- limit groups [37] (otherwise known as fully residually free groups), and more generally groups that act freely on $\mathbb{R}^{n}$-trees (cf. [2], [9], [20]).

A great deal is known about toral relatively hyperbolic groups. In particular, the isomorphism problem is solvable in this class [10]. They are all Hopfian [19]. Degenerate examples such as $\mathbb{Z}$ are not finitely co-Hopfian, but the following result shows that this is the only obstruction to being cofinitely Hopfian.

Theorem 3.1. Let $\Gamma$ be a toral relatively hyperbolic group and let $\varphi: \Gamma \rightarrow \Gamma$ be a homomorphism so that $\varphi(\Gamma)$ has finite index in $\Gamma$. Then $\varphi$ is an injection.

Proof. In order to obtain a contradiction we suppose that $[\Gamma: \varphi(\Gamma)]<\infty$ and that $\varphi$ is not an injection. Since $\Gamma$ is torsion-free and each $\varphi^{i}(\Gamma)$ has finite index in $\Gamma$, the groups $K_{i}=\operatorname{ker}(\varphi) \cap \varphi^{i}(\Gamma)$ are all non-trivial. Consequently, the kernels $\operatorname{ker}(\varphi) \subset \operatorname{ker}\left(\varphi^{2}\right) \subset \cdots$ are all distinct, so there is a properly descending sequence of epimorphisms

$$
\Gamma \rightarrow \varphi(\Gamma) \rightarrow \varphi^{2}(\Gamma) \rightarrow \cdots
$$


But [19], Theorem 5.2, tells us that such sequences do not exist. (To see that [19], Theorem 5.2, applies, note that each $\phi^{i}(\Gamma)$, being a finitely generated subgroup of $\Gamma$, is a $\Gamma$-limit group.) This contradiction implies that $\varphi$ must in fact be an injection.

Corollary 3.2. Let $\Gamma$ be a co-Hopfian toral relatively hyperbolic group. Then $\Gamma$ is cofinitely Hopfian.

\section{Lattices}

The discussions leading to this paper began with a desire to extend the results of [7] concerning convex cocompact Kleinian groups to non-uniform lattices and geometrically finite groups. Corollary 3.2 represents progress in this direction since it applies to the fundamental groups of finite volume hyperbolic manifolds whose cusp cross-sections are tori. However, non-uniform lattices whose cusp cross-sections are more general flat manifolds need not be toral relatively hyperbolic (though they will always have a subgroup of finite index that is), so are not covered by Corollary 3.2. To circumvent this difficulty we use the following result of Hirshon [22] instead of Theorem 3.1 (cf. [7], Proposition 4.4). Hirshon's argument is a variant on the standard argument for the Hopficity of residually finite groups.

Lemma 4.1. Let $\Gamma$ be a finitely generated residually finite group with no non-trivial finite normal subgroup. If $\varphi: \Gamma \rightarrow \Gamma$ is an endomorphism with image of finite index then $\varphi$ is a monomorphism.

Although our initial interest lay with lattices in $\mathrm{SO}(n, 1)$, it does not seem any harder to deal with similar lattices in more general Lie groups. We would like to have given an elementary proof ${ }^{1}$ covering a class of lattices that includes those in $\mathrm{SO}(n, 1)$. However, we are unable to find a proof using less machinery than what follows. (Some alternative approaches are discussed in the next section.)

Proposition 4.2. Let $G$ be a connected semisimple Lie group with trivial centre and no compact factors, and let $\Gamma$ be a lattice in $G$. If either

(1) $\Gamma$ is cocompact or

(2) $\Gamma$ is irreducible

then it is cofinitely Hopfian.

Proof. Since $G$ is connected and centreless, the adjoint representation is faithful and $G$ is linear. Also, $\Gamma$ is finitely generated (see, for example, [28], chap. IX, (3.1) (ii), p. 311), and Mal'cev famously observed that finitely generated linear groups are

\footnotetext{
${ }^{1}$ We thank Nicolas Monod for his thoughts on this.
} 
residually finite [27]. Moreover $\Gamma$ has no non-trivial finite normal subgroup (this follows from Borel's Density Theorem; see [48], Corollary 4.42). Thus we are in the situation of Lemma 4.1: if the image of $\varphi: \Gamma \rightarrow \Gamma$ has finite index then $\varphi$ is injective.

The group $\Gamma$ has a torsion-free subgroup $H$ of finite index, by Selberg's Lemma ([38]; see also [48], [Theorem 4.60]). Let $H_{k}=\varphi^{-k}(H)$ for $k \geq 0$. Then [ $\Gamma$ : $\left.H_{k}\right] \leq[\Gamma: H]$ and $H_{k}$ is torsion-free since $\varphi$ is injective for all $k \geq 0$. Since $\Gamma$ is finitely generated it has only finitely many subgroups of index bounded by [ $\Gamma: H]$. Therefore there are integers $m, n>0$ such that $H_{m+n}=H_{n}$, and hence $\varphi^{m}\left(H_{n}\right) \leq H_{n}$. If $\Gamma$ is cocompact then so is $H_{n}$ and in this case $\varphi^{m}\left(H_{n}\right)=H_{n}$, by Theorem 2 of [33] (or by using any suitable volume-type invariant, in particular Löh and Sauer's Lipschitz simplicial volume [26]). Since $\Gamma$ has no non-trivial finite normal subgroup, it follows that $\varphi^{m}(\Gamma)=\Gamma$ also, and hence $\varphi$ is an automorphism.

If $\Gamma$ is irreducible and $G$ is not $\operatorname{PSL}(2, \mathbb{R})$ the result follows from Mostow-Prasad rigidity [32], Theorem B. If $G=\operatorname{PSL}(2, \mathbb{R})$ then $\Gamma$ is either cocompact or virtually free, and we may use multiplicativity of the rational Euler characteristic, as indicated in the next section.

Added in proof: Since writing this article we learned of the existence of [24] in which Humphreys and Johnson study the finite co-Hopf property for lattices. The first paragraph of the preceding proof reduces Proposition 4.2 to a special case of their main theorem.

Products of irreducible lattices can be dealt with by combining the above argument with consideration of the structure of centralisers. And in the higher rank case, far more general results are implied by Margulis super-rigidity [28].

Results such as Proposition 4.2 allow one to extend the range of maps in classical rigidity theorems. For example:

Corollary 4.3. Let $M_{1}$ and $M_{2}$ be finite volume hyperbolic $n$-manifolds, $n \geq 3$. Suppose there are proper open surjective maps $f: M_{1} \rightarrow M_{2}$ and $g: M_{2} \rightarrow M_{1}$. Then $M_{1}$ and $M_{2}$ are isometric.

Proof. The compositions $h=g \circ f: M_{1} \rightarrow M_{1}$ and $h^{\prime}=f \circ g: M_{2} \rightarrow M_{2}$ are proper, open and surjective. Thus by Proposition $2.1, h_{*} \pi_{1}\left(M_{1}\right)$ has finite index in $\pi_{1}\left(M_{1}\right)$ and similarly for $h_{*}^{\prime}$. Proposition 4.2(2) tells us that both $h_{*}$ and $h_{*}^{\prime}$ are in fact isomorphisms. Hence $f_{*}$ and $g_{*}$ are isomorphisms and Mostow rigidity applies.

Corollary 4.3 applies in particular to hyperbolic knot complements. Knot complements will be studied in more detail in Section 7. 


\section{Various Hopf properties and volume-type invariants}

There are several more notions related to the Hopf property that we should consider. A group $\Gamma$ is finitely co-Hopfian if every injective endomorphism with image of finite index is an automorphism. It is (finitely) hyper-Hopfian if every endomorphism $\varphi$ such that $\varphi(\Gamma)$ is a normal subgroup with (finite) cyclic quotient $\Gamma / \varphi(\Gamma)$ is an automorphism. Next, we say that $\Gamma$ satisfies the volume condition if isomorphic subgroups of finite index necessarily have the same index. Finally, we say that $\Gamma$ satisfies the rank condition if any proper subgroup of finite index requires strictly more generators that $\Gamma$ does.

Only a little argument is needed to see that if $\Gamma$ is cofinitely Hopfian, then $\Gamma$ is finitely hyper-Hopfian and finitely co-Hopfian; that finitely hyper-Hopfian groups are Hopfian; and that groups satisfying the volume condition or the rank condition are finitely co-Hopfian. As recalled earlier, residually finite groups are Hopfian.

A useful example to keep in mind is $\mathbb{Z}_{2}=\mathbb{Z} / 2 \mathbb{Z}$, which is Hopfian and co-Hopfian but not finitely hyper-Hopfian.

Non-abelian free groups of finite rank and hyperbolic surface groups satisfy both the volume condition and the rank condition, by multiplicativity of the Euler characteristic, while irreducible lattices in semi-simple Lie groups satisfy the volume condition but in general do not satisfy the rank condition (consideration of hyperbolic 3-manifolds that fibre over the circle is enough to prove this).

Less obviously, Reznikov [33] defined several "volume-type" invariants of groups which take values in $[0, \infty)$ and are multiplicative on passing to subgroups of finite index: if $\Gamma_{0}<\Gamma$ is a subgroup of index $d$ then the invariant of $\Gamma_{0}$ is $d$ times that of $\Gamma$. (These are called multiplicative invariants in [24].) He showed that one such invariant, the "rank volume" $V_{r}(\Gamma)$ is strictly positive if $\Gamma$ has a presentation of deficiency greater than 1 , and that $V_{r}(\Gamma) \geq V_{r}(\Gamma / H)$ for any $H \unlhd \Gamma$. It follows that every group with deficiency greater than 1 satisfies the volume condition, and that it is cofinitely Hopfian if it is Hopfian.

A more classical example of a volume-type invariant is the rational Euler characteristic. We recall that if a group $G$ has a subgroup $H$ of finite index that has a compact classifying space $K(H, 1)$, then the rational Euler characteristic $\chi^{\text {virt }}(\Gamma):=$ $[G: H]^{-1} \chi(H)$ is well defined and multiplicative. Borel and Serre [5] proved that lattices in semisimple Lie groups have a well-defined rational Euler characteristic.

Thinking of the rational Euler characteristic in topological terms, one sees that it is an example of a volume-type invariant that arises in the following way: one has a class of spaces (or orbispaces) on which an invariant is defined; each space is defined uniquely up to some notion of equivalence (e.g. homotopy equivalence or homeomorphism) by its fundamental group, and the invariant is constant on equivalence classes; the class is closed under passage to finite-sheeted covers and the invariant multiplies by the index on passage to a finite-sheeted cover. If a group $G$ is torsionfree, residually finite and is the fundamental group of a space in the class that has non-zero invariant, then $G$ is cofinitely Hopfian. 
Gromov's simplicial volume [18] fulfills these conditions in classes of manifolds that are determined up to homeomorphism by their fundamental group. Classes of manifolds satisfying this condition and for which this volume is positive include compact locally-symmetric spaces of non-compact type and of dimension $\neq 4$ [13], [25]. Löh and Sauer's notion of Lipschitz simplicial volume is a useful variant on Gromov's definition: this is non-zero in interesting cases where the simplicial volume vanishes, for example certain of the non-compact locally symmetric spaces that arise in the context of our Proposition 4.2. In particular, Theorem 1.5 and Corollary 1.6 of [26] imply that the fundamental group of any locally symmetric space of noncompact type is cofinitely Hopfian.

\section{Free-by-cyclic groups}

Finitely presented free-by-cyclic groups have received a great deal of attention in recent years in part because they form a rich context in which to draw out distinctions between the different notions of non-positive curvature in group theory (cf. [6]). In this section we shall determine which of these groups are cofinitely Hopfian.

Notation: In what follows we let $\Gamma^{\prime}, Z \Gamma$ and $\operatorname{cd}(\Gamma)$ denote the commutator subgroup, centre and cohomological dimension, respectively, of the group $\Gamma$. Let $F_{r}$ denote the free group of rank $r$.

We need the following two lemmas.

Lemma 6.1. Let $\Gamma=F_{r} \rtimes \mathbb{Z}$ and suppose that $\varphi: \Gamma \rightarrow \Gamma$ is an endomorphism such that $N=\varphi(\Gamma)$ is normal in $\Gamma$ and $\Gamma / N \cong \mathbb{Z}$. Then either $\Gamma \cong F_{r} \times \mathbb{Z}$ or $\Gamma \cong \mathbb{Z} \rtimes_{-1} \mathbb{Z}$.

Proof. The non-trivial assertion in the lemma concerns the case $r \geq 2$. Thus we assume that $r \geq 2$ and that $\Gamma$ has no decomposition $F_{\rho} \rtimes \mathbb{Z}$ with $\rho<r$.

The group $\Gamma$ is coherent [14], and so the finitely generated subgroup $N$ is finitely presentable, and $\operatorname{cd}(N) \leq \operatorname{cd}(\Gamma)=2$. Therefore $\operatorname{cd}(N)=\operatorname{cd}(\Gamma)-\operatorname{cd}(\mathbb{Z})=1$, by Theorem 5.6 of [4]. Hence $N$ is also free, of finite rank $s$, say. Clearly $s \geq r$, by minimality of $r$.

Let $p: \Gamma \rightarrow \mathbb{Z}$ be an epimorphism with kernel $F \cong F_{r}$. The image of $F$ in $N$ is a finitely generated normal subgroup. Therefore either $\varphi(F)=1$ or $\varphi(F)$ has finite index in $N$. If $\varphi(F)=1$ then $N$ is cyclic, contrary to the hypothesis $r>1$. Hence $[N: \varphi(F)]$ is finite, and so $\varphi(F)$ is free of finite rank $t$, say. Now $\chi(\varphi(F))=$ $1-t=[N: \varphi(F)] \chi(N)=[N: \varphi(F)](1-s) \neq 0$, and $t \leq r \leq s$ since $\varphi(F)$ is a quotient of $F$. Hence $\varphi(F)=N$ and $t=s$, so $s=r$. Therefore $\left.\varphi\right|_{F}: F \rightarrow N$ is an isomorphism, by the Hopficity of $F_{r}$. Let $q=\left(\left.\varphi\right|_{F}\right)^{-1} \circ \varphi: \Gamma \rightarrow F$. Then $(q, p): \Gamma \rightarrow F \times \mathbb{Z}$ is an isomorphism.

The groups $\Gamma \cong F_{r} \times \mathbb{Z}$ and $\Gamma \cong \mathbb{Z} \rtimes_{-1} \mathbb{Z}$ are residually finite but are not finitely hyper-Hopfian. 
Lemma 6.2. If $\theta \in \operatorname{Out}\left(F_{r}\right)$ has infinite order, then the set of integers $\{n \mid$ there exists $\psi$ with $\left.\psi^{n}=\theta\right\}$ is finite.

Proof. This is an immediate consequence of a theorem of Emina Alibegović [1]. Her result is cast in the language of translation lengths. Recall that the translation length $\tau(g)$ of an element $g$ in a group $G$ with finite generating set $A$ is defined to be $\lim _{m} d\left(1, g^{m}\right) / m$, where $d$ is the word metric associated to $A$. Since $\tau\left(g^{n}\right)=$ $|n| \tau(g)$ for all $n \in \mathbb{Z}$, the lemma would be proved if we knew that there was a positive constant $\epsilon_{r}>0$ such that $\tau(\theta)>\epsilon_{r}$ for all $\theta \in \operatorname{Out}\left(F_{r}\right)$ of infinite order. And this is exactly what Alibegović proves.

Theorem 6.3. Let $\Gamma=F_{r} \rtimes_{\theta} \mathbb{Z}$. Then the following are equivalent:

(1) $\Gamma$ is cofinitely Hopfian;

(2) $\Gamma$ is finitely co-Hopfian;

(3) $\Gamma$ is hyper-Hopfian;

(4) $\Gamma$ is finitely hyper-Hopfian;

(5) $\Gamma$ has trivial centre.

Proof. The theorem is obvious if $r=1$, so we assume that $r \geq 2$ is minimal among the ranks of all free normal subgroups of $\Gamma$ with cyclic quotient.

The implications (1) $\Longrightarrow(2),(1) \Longrightarrow$ (4) and (3) $\Longrightarrow$ (4) are clear, while (2) $\Longrightarrow$ (1) follows from Lemma 4.1 since $\Gamma$ is finitely generated, residually finite and torsion free. The implication (4) $\Longrightarrow$ (3) follows from Lemma 6.1 since neither $\Gamma \cong F_{r} \times \mathbb{Z}$ nor $\Gamma \cong \mathbb{Z} \rtimes_{-1} \mathbb{Z}$ is finitely hyper-Hopfian.

Let $[\theta]$ be the image of $\theta$ in $\operatorname{Out}\left(F_{r}\right)$ and note that $Z \Gamma=1$ if and only if $[\theta]$ has infinite order. Suppose that $[\theta]^{d}=1$ for some finite $d>0$. If $s \equiv 1 \bmod d$, then $\left[\theta^{s}\right]=[\theta]$, and so $\Gamma \cong F_{r} \rtimes_{\theta^{s}} \mathbb{Z}$, which is isomorphic to a normal subgroup of index $s$ in $\Gamma$, with quotient $\mathbb{Z} / s \mathbb{Z}$. Thus $\pi$ is not finitely hyper-Hopfian, and so (4) $\Longrightarrow(5)$.

We shall show that (4) $\Longrightarrow$ (2) in the course of showing that (5) $\Longrightarrow$ (2). Suppose that $\varphi$ is an injective endomorphism of $\Gamma=F_{r} \rtimes_{\theta} \mathbb{Z}$ with image $N$ of finite index $d$. Let $M=\varphi\left(F_{r}\right)$. Let $\Gamma^{\tau}<\Gamma$ be the preimage of the torsion subgroup of $\Gamma^{a b}=\Gamma / \Gamma^{\prime}$. Then $\Gamma^{\prime} \leq \Gamma^{\tau}$ and $\Gamma / \Gamma^{\tau} \cong \mathbb{Z}^{\beta}$. The image of $N / N^{\tau} \cong \mathbb{Z}^{\beta}$ in $\Gamma / \Gamma^{\tau}$ has finite index since $[\Gamma: N]<\infty$. Therefore there is an epimorphism $g: \Gamma \rightarrow \mathbb{Z}$ such that $M=N \cap \operatorname{ker}(g)$. In particular, $M$ has finite index in $\operatorname{ker}(g)$. Hence $M=\operatorname{ker}(g)$, by minimality of $r$, and so $N$ is normal in $\Gamma$ and $\Gamma / N \cong \mathbb{Z} / d \mathbb{Z}$. Thus (4) $\Longrightarrow$ (2), and we have proved that the first four conditions are equivalent.

Continuing with the notation of the previous paragraph, we have $\Gamma / M \cong \mathbb{Z}$. Fix $t \in \Gamma$ such that $g(t)$ generates $G / M$. Conjugation by $t$ induces an automorphism $\psi \in \operatorname{Aut}(M)$. Then $N$ is generated by $M$ and $t^{d}$ and $\varphi$ induces an isomorphism from $\Gamma=F_{r} \rtimes_{\theta} \mathbb{Z}$ to $N=M \rtimes_{\psi^{d}} \mathbb{Z}$ that is compatible with the given semidirect product decompositions. It follows that the image of $\phi$ is conjugate to the image of $\psi^{d}$ in $\operatorname{Out}\left(F_{r}\right)$. By considering iterates of $\varphi$, we conclude that the image of $\theta$ has a 
$d^{n}$-root in $\operatorname{Out}\left(F_{r}\right)$ for all positive integers $n$. By Lemma 6.2, it follows that $\theta$ has finite order. Thus (5) $\Longrightarrow$ (2). This completes the proof.

If $\Gamma / \Gamma^{\tau} \cong \mathbb{Z}$ then the preceding proof can be simplified. First, the implication (4) $\Longrightarrow$ (1) follows from the observation that if $\Gamma=N \rtimes_{\theta} \mathbb{Z}$ where $N$ is cofinitely Hopfian and $\varphi: \Gamma \rightarrow \Gamma$ is an endomorphism with image of finite index and $\varphi(N) \leq$ $N$, then $\varphi$ is a monomorphism onto a normal subgroup and $\Gamma / \varphi(\Gamma)$ is finite cyclic. And in proving (5) $\Longrightarrow$ (2) one does not need Lemma 6.2.

Remark 6.4. The conditions listed in the preceding theorem do not imply that $\Gamma$ is co-Hopfian. For instance, if $K$ is a composite of fibred knots or is an algebraic knot other than a torus knot and $\Gamma$ is the fundamental group of $\mathbb{S}^{3} \backslash K$, then $\Gamma / \Gamma^{\prime} \cong \mathbb{Z}$, $\Gamma^{\prime}$ is free and $Z \Gamma=1$, but $\Gamma$ is not co-Hopfian [17].

Let $G=F *_{\theta}$ be an ascending HNN extension, where $F=F_{r}$ for some $r>1$ and $\theta: F \rightarrow F$ is a monomorphism with image a proper subgroup. Then $G$ has trivial centre and is Hopfian [16]. Is it cofinitely Hopfian? Lemma 6.1 may be adapted to exclude such groups, while the implications (1) $\Leftrightarrow(2),(1) \Longrightarrow(4)$ and (3) $\Longrightarrow$ (4) of Theorem 6.3 hold. Does (4) $\Longrightarrow$ (2)? (If $r=1$ then $G$ is a solvable Baumslag-Solitar group, which is not finitely hyper-Hopfian.)

\section{3-manifolds and knot groups}

Let $M$ be a compact orientable 3-manifold and let $\pi=\pi_{1}(M)$. Then $M$ is Haken, hyperbolic or Seifert fibred, as a consequence of Thurston's Geometrisation Conjecture, now proven by Perelman. In each case $\pi$ is residually-finite and hence Hopfian [21], [42]. If $M$ is closed then $\pi$ is co-Hopfian if and only if $M$ is irreducible and has no finite cover which is a direct product of a (closed) surface with $\mathbb{S}^{1}$ or a torus bundle over $\mathbb{S}^{1}$ [46]. If $M$ is irreducible and has nonempty toral boundary then $\pi$ is co-Hopfian if and only if it is not $\mathbb{Z}^{2}$ and no non-trivial Seifert fibred piece of the JSJ decomposition of $M$ meets $\partial M$, by Theorem 2.5 of [17]. The group $\pi$ satisfies the volume condition (as defined in Section 5 above) if and only if it is either a proper free product other than $\mathbb{Z} / 2 * \mathbb{Z} / 2$ or if $M$ is irreducible and has no finite cover which is a direct product of a surface with $\mathbb{S}^{1}$ or a torus bundle over $\mathbb{S}^{1}$ [46], [49].

From these results we see: if $K$ is a knot in $\mathbb{S}^{3}$, then the knot group $\pi_{K}$ is Hopfian; it is co-Hopfian if and only if $K$ is not a torus knot, cable knot or composite knot; and it satisfies the volume condition (and is finitely co-Hopfian) if and only if $K$ is not a torus knot. (See Corollaries 2.6 and 7.5 of [17], [49] and Section 7.1 below.) The following theorem provides the complementary classification for the cofinite Hopf property.

We note also the following standard facts from knot theory. Let $K$ be a knot with group $\pi=\pi_{K}$. Then the abelianization $\pi / \pi^{\prime}$ is infinite cyclic. If $K$ is non-trivial 
then the image of the fundamental group of a Seifert surface of minimal genus is a non-abelian free subgroup of $\pi$. In particular, the group of a fibred knot is free-bycyclic. Torus knots are fibred and their groups have infinite cyclic centres. (See, for example, [34].)

Theorem 7.1. Let $K$ be a knot in $\mathbb{S}^{3}$. The knot group $\pi_{K}$ is cofinitely Hopfian if and only if $K$ is not a torus knot.

Proof. If $K$ is a torus knot then $\pi_{K}^{\prime}$ is a finitely generated free group and $Z \pi_{K} \neq 1$. Therefore $\pi_{K}$ is not cofinitely Hopfian, by Theorem 6.3. (A more explicit proof is given below.)

Conversely, if $K$ is not a torus knot then $\pi_{K}$ is finitely co-Hopfian [17]. Since $\pi_{K}$ is finitely generated, residually finite and torsion free Lemma 4.1 then implies that $\pi_{K}$ is cofinitely Hopfian.

We recover the following result of [39].

Corollary 7.2. Let $K$ be a knot in $\mathbb{S}^{3}$. Then $\pi_{K}$ is hyper-Hopfian if and only if $K$ is not a torus knot.

Proof. Let $\pi=\pi_{K}$ and assume that $K$ is not a torus knot. Then $K$ is non-trivial and $\pi$ is non-abelian. Let $\varphi: \pi \rightarrow \pi$ be an endomorphism with $\varphi(\pi)$ a normal subgroup of $\pi$ and $\pi / \varphi(\pi)$ cyclic. Then $\pi^{\prime} \leq \varphi(\pi)$ since knot groups have abelianization $\mathbb{Z}$. If $\varphi(\pi)=\pi^{\prime}$, then $\pi^{\prime}$ is finitely generated. But then $K$ is fibred and so $\pi^{\prime}$ is free [41]. Since $\pi$ and $\varphi(\pi)$ have cyclic abelianization this is only possible if $\pi \cong \mathbb{Z}$, which is contrary to our assumption. Therefore $\pi / \varphi(\pi)$ is finite, and so $\varphi$ is an automorphism by Theorem 7.1.

The complement of any knot in $\mathbb{S}^{3}$ is aspherical [31] and hence is determined up to homotopy equivalence by its fundamental group. But, famously, the fundamental group does not determine the knot exterior up to homeomorphism. For example, the granny knot (the sum of two copies of the left hand trefoil knot) and the reef knot (the sum of a trefoil knot and its reflection) have the same group, but their exteriors are not homeomorphic. On the other hand, Waldhausen [44] proved that any homotopy equivalence of knot complements that preserves the peripheral structure is homotopic to a homeomorphism. More precisely, he shows that if $X$ is the complement of an open regular neighbourhood of a knot $K$, then any homotopy equivalence of the pair $(X, \partial X)$ is homotopic to a homeomorphism (and it is easy to extend this to the whole of $\left.\mathbb{S}^{3} \backslash K\right)$.

Theorem 7.3. Let $K$ be a knot that is not a torus knot and let $f$ be a proper open self-map of the knot complement $\mathbb{S}^{3} \backslash K$. Then $f$ is homotopic to a homeomorphism. 
Proof. Since $f$ is proper and open and $\mathbb{S}^{3} \backslash K$ is connected, $f$ is surjective. Therefore $n=\left[\pi_{K}: f_{*}\left(\pi_{K}\right)\right]$ is finite, by Proposition 2.1. Since $K$ is not a torus knot, Theorem 7.1 tells us that $f_{*}$ is an isomorphism. And since knot complements are aspherical, it follows that $f$ is a homotopy equivalence. By the work of Waldhausen quoted above, we will be done provided that we can argue that $f$ preserves the peripheral structure of the knot. For the benefit of readers who are not specialists in 3-manifold theory, we explain why this is true with a proof that has a group-theoretic flavour.

Let $N \cong \mathbb{S}^{1} \times \mathbb{D}^{2}$ be a closed regular neighbourhood of $K$, where $\mathbb{D}$ is the open unit disc in $\mathbb{R}^{2}$. Let $r \mathbb{D}$ be the disc of radius $r \in(0,1]$, let $r N$ be the corresponding neighourhood of $K$ and let $U_{r}=r N \backslash K$. The closure $X$ of $\mathbb{S}^{3} \backslash N$ is a compact manifold with boundary $\partial X$ a torus, and $X \hookrightarrow \mathbb{S}^{3} \backslash K$ is a homotopy equivalence. Adjusting $f$ by a homotopy if necessary, we may assume that $f(X) \subseteq X$. Moreover, since $f$ is proper, we may assume that it maps each $U_{r}$ inside a suitable $U_{r^{\prime}}$ (since the sets $U_{r}$ form a cofinal system of closed neighbourhoods of the single end of the noncompact 3-manifold $\mathbb{S}^{3} \backslash K$ ). Therefore, adjusting by a radial projection, we can arrange that $f(\partial X) \subset \partial X$. We fix a basepoint $x_{0} \in \partial X$ and homotope $f$ so that $f\left(x_{0}\right)=x_{0}$.

Since $K$ is non-trivial, $\pi_{1}\left(\partial X, x_{0}\right) \rightarrow \pi_{K}=\pi_{1}\left(\mathbb{S}^{3} \backslash K, x_{0}\right)$ is injective (cf. [31]); let $P$ denote its image. We will be done if we can prove that $\left.f\right|_{\partial X}$ induces an isomorphism $P \rightarrow P$.

Since $f_{*}$ is injective, $f_{*}^{-1}(P) \cong \mathbb{Z}^{2}$, so if $f_{*}(P)$ were not the whole of $P$ then there would be elements of $\pi_{K} \backslash P$ that commuted with $P$. But then $\Gamma=\pi_{K} *_{P} \pi_{K}$ would contain a copy of $\mathbb{Z}^{3}$, and $\Gamma$ is the fundamental group of the closed, aspherical 3-manifold $Y$ obtained by doubling $X$ along its boundary. Since infinite coverings of 3-manifolds collapse to 2-complexes, $\mathbb{Z}^{3}$ cannot be the fundamental group of an infinite-sheeted covering of $Y$, so $\pi_{1}(Y)$ would be virtually abelian. But this is absurd since $\pi_{1}(Y)$ contains $\pi_{K}$ and non-trivial knot groups have non-abelian free subgroups. This contradiction completes the proof.

Corollary 7.4. Let $K$ be a knot and let $f: \mathbb{S}^{3} \rightarrow \mathbb{S}^{3}$ be an open mapping of finite degree not equal to 1 such that $f^{-1}(K)=K$. Then $K$ is a torus knot.

Proof. The map $f$ is surjective since it is open and $\mathbb{S}^{3}$ is compact. Therefore $f(K)=$ $K$ and the restriction $f: \mathbb{S}^{3} \backslash K \rightarrow \mathbb{S}^{3} \backslash K$ is proper, open and surjective. Since the degree of $f$ is not $1, f$ cannot be homotopic to a homeomorphism. Thus Theorem 7.3 implies $K$ is a torus knot.

A map is said to be discrete if the preimage of each point in the target is a discrete set.

Corollary 7.5. Let $K$ be a non-torus knot and let $f: \mathbb{S}^{3} \rightarrow \mathbb{S}^{3}$ be a discrete open mapping such that $f^{-1}(K)=K$. Then $f$ is a homeomorphism. 
Proof. A discrete open mapping homotopic to a homeomorphism is a homeomorphism - see [8], [43].

Notice, with regard to the context of [7], that the above corollary implies that the branch set of a quasiregular map cannot be a completely invariant non-torus knot. The assumption here that the mapping is discrete (as opposed to light) is necessary due to the counterexamples constructed in [47].

7.1. Torus knots are not cofinitely Hopfian. The fundamental group of a torus knot has the form

$$
\Gamma_{m, n}=\left\langle a, b \mid a^{n}=b^{m}\right\rangle .
$$

The centre of such a group is infinite cyclic, generated by $z:=a^{n}=b^{m}$. Let $Q \cong(\mathbb{Z} / n) *(\mathbb{Z} / m)$ be the quotient by the centre.

We consider maps $\varphi: \Gamma_{m, n} \rightarrow \Gamma_{m, n}$ of the form

$$
\varphi(a)=a z^{p} \quad \text { and } \quad \varphi(b)=b z^{q} .
$$

This formula defines a homomorphism if and only if $\varphi\left(a^{n}\right)=\varphi\left(b^{m}\right)$, that is, $n p=$ $m q$ (which we write as $r-1$ ). The image of $\varphi$ is of finite index because it maps onto $Q$ and intersects $Z=\langle z\rangle$ non-trivially. The normal form theorem for amalgamated free products shows that $\varphi$ is injective. Since no commutators lie in the kernel, $\varphi^{-1}(Z)=Z$. And since $\varphi(z)=\varphi\left(a^{n}\right)=z^{r}$, we see that $\varphi$ is not onto unless $p=q=0$. Thus we have proved:

Proposition 7.6. If $\Gamma_{m, n}=\left\langle a, b \mid a^{n}=b^{m}\right\rangle$ and $r$ is an integer, with $r \equiv$ $1 \bmod n$ and $r \equiv 1 \bmod m$, then $\left[\varphi(a):=a^{r}, \varphi(b):=b^{r}\right]$ defines a monomorphism $\varphi: \Gamma_{m, n} \rightarrow \Gamma_{m, n}$ whose image has finite index. But $\varphi$ is onto only if $r= \pm 1$.

\section{References}

[1] E. Alibegović, Translation lengths in $\operatorname{Out}\left(F_{n}\right)$. Geom. Dedicata 92 (2002), 87-93. Zbl 1041.20024 MR 1934012

[2] E. Alibegović, A combination theorem for relatively hyperbolic groups. Bull. London Math. Soc. 37 (2005), 459-466. Zbl 1074.57001 MR 2131400

[3] G. Baumslag and D. Solitar, Some two-generator one-relator non-Hopfian groups. Bull. Amer. Math. Soc. 68 (1962), 199-201. Zbl 0108.02702 MR 0142635

[4] R. Bieri, Homological dimension of discrete groups. Queen Mary College Mathematical Notes, Queen Mary College Department of Pure Mathematics, London 1976. Zbl 0357.20027 MR 0466344

[5] A. Borel and J.-P. Serre, Corners and arithmetic groups. Comment. Math. Helv. 48 (1973), 436-491. Zbl 0274.22011 MR 0387495 
[6] M. R. Bridson, Non-positive curvature and complexity for finitely presented groups. In Proc. Internat. Congr. of Mathematicians. Vol. II, Eur. Math. Soc., Zürich 2006, 961-987. Zbl 1108.20041 MR 2275631

[7] M. Bridson, A. Hinkkanen, and G. Martin, Quasiregular self-mappings of manifolds and word hyperbolic groups. Compos. Math. 143 (2007), 1613-1622. Zbl 1131.20031 MR 2371385

[8] A. V. Černavskiǔ, Finite-to-one open mappings of manifolds. Mat. Sb. (N.S.) 65 (107) (1964), 357-369; English transl. Amer. Math. Soc. Transl. Ser. (2) 100 (1972), 253-267. Zbl 0129.15003 MR 0172256

[9] F. Dahmani, Combination of convergence groups. Geom. Topol. 7 (2003), 933-963. Zbl 1037.20042 MR 2026551

[10] F. Dahmani and D. Groves, The isomorphism problem for toral relatively hyperbolic groups. Publ. Math. Inst. Hautes Études Sci. 107 (2008), 211-290. Zbl 05645799 MR 2434694

[11] R. J. Daverman, Hyperhopfian groups and approximate fibrations. Compositio Math. 86 (1993), 159-176. Zbl 0788.57012 MR 1214455

[12] T. Delzant and L. Potyagailo, Endomorphisms of Kleinian groups. Geom. Funct. Anal. 13 (2003), 396-436. Zbl 1108.20047 MR 1982149

[13] F. T. Farrell and L. E. Jones, Topological rigidity for compact nonpositively curved manifolds. In Differential geometry: Riemannian geometry (Los Angeles, CA, 1990), Proc. Sympos. Pure Math. 54, Amer. Math. Soc., Providence, RI 1993, 229-274. Zbl 0796.53043 MR 1216623

[14] M. Feighn and M. Handel, Mapping tori of free group automorphisms are coherent. Ann. of Math. (2) 149 (1999), 1061-1077. Zbl 0938.20022 MR 1709311

[15] F. W. Gehring and G. J. Martin, Discrete quasiconformal groups, I. Proc. London Math. Soc. (3) 55 (1987), 331-358. Zbl 0628.30027 MR 896224

[16] R. Geoghegan, M. L. Mihalik, M. Sapir, and D. T. Wise, Ascending HNN extensions of finitely generated free groups are Hopfian. Bull. London Math. Soc. 33 (2001), 292-298. Zbl 1028.20020 MR 1817768

[17] F. González-Acuña and W. C. Whitten, Imbeddings of three-manifold groups. Mem. Amer. Math. Soc. 99 (1992), no. 474. Zbl 0756.57002 MR 1117167

[18] M. Gromov, Volume and bounded cohomology. Inst. Hautes Études Sci. Publ. Math. 56 (1982), 5-99. Zbl 0516.53046 MR 686042

[19] D. Groves, Limit groups for relatively hyperbolic groups II: Makanin-Razborov diagrams. Geom. Topol. 9 (2005), 2319-2358. Zbl 1100.20032 MR 2209374

[20] V. Guirardel, Limit groups and groups acting freely on $\mathbb{R}^{n}$-trees. Geom. Topol. 8 (2004), 1427-1470. Zbl 1114.20013 MR 2119301

[21] J. Hempel, Residual finiteness for 3-manifolds. In Combinatorial group theory and topology (Alta, Utah, 1984), Ann. of Math. Stud. 111, Princeton Univ. Press, Princeton, NJ, 1987, 379-396. Zbl 0772.57002 MR 0895623

[22] R. Hirshon, Some properties of endomorphisms in residually finite groups. J. Austral. Math. Soc. Ser. A 24 (1977), 117-120. Zbl 0374.20043 MR 0470080 
[23] H. Hopf, Beiträge zur Klassifizierung der Flächenabbildungen. J. Reine Angew. Math. 165 (1931), 225-236. JFM 57.0726.01 Zbl 0002.05602

[24] J. J. A. M. Humphreys and F. E. A. Johnson, Multiplicative invariants and the finite co-Hopfian property. Mathematika 55 (2009), 115-127. Zbl 05666967 MR 2573604

[25] J.-F. Lafont and B. Schmidt, Simplicial volume of closed locally symmetric spaces of non-compact type. Acta Math. 197 (2006), 129-143. Zbl 1111.57020 MR 2285319

[26] C. Löh and R. Sauer, Degree theorems and Lipschitz simplicial volume for nonpositively curved manifolds of finite volume. J. Topol. 2 (2009), 193-225. Zbl 1187.53043 MR 2499443

[27] A. I. Mal'cev, On the faithful representation of infinite groups by matrices. Mat. Sb. (N.S.) 8 (50) (1940), 405-422; English transl. Amer. Math. Soc. Transl. Ser. (2) 45 (1965), 1-18. Zbl 0158.02905 MR 0003420

[28] G. A. Margulis, Discrete subgroups of semisimple Lie groups. Ergeb. Math. Grenzgeb.(3) 17, Springer-Verlag, Berlin 1991. Zbl 0732.22008 MR 1090825

[29] G. Martin, V. Mayer, and K. Peltonen, The generalized Lichnerowicz problem: uniformly quasiregular mappings and space forms. Proc. Amer. Math. Soc. 134 (2006), 2091-2097. Zbl 1094.30029 MR 2215779

[30] G. D. Mostow, Quasi-conformal mappings in $n$-space and the rigidity of hyperbolic space forms. Inst. Hautes Études Sci. Publ. Math. 34 (1968), 53-104. Zbl 0189.09402 MR 0236383

[31] C. D. Papakyriakopoulos, On Dehn's lemma and the asphericity of knots. Ann. of Math. (2) 66 (1957), 1-26. Zbl 0078.16402 MR 0090053

[32] G. Prasad, Strong rigidity of Q-rank 1 lattices. Invent. Math. 21 (1973), 255-286. Zbl 0264.22009 MR 0385005

[33] A. Reznikov, Volumes of discrete groups and topological complexity of homology spheres. Math. Ann. 306 (1996), 547-554. Zbl 0859.20027 MR 1415078

[34] D. Rolfsen, Knots and links. Mathematics Lecture Series 7, Publish or Perish, Berkeley, Calif., 1976, Zbl 0339.55004 MR 0515288

[35] Z. Sela, Structure and rigidity in (Gromov) hyperbolic groups and discrete groups in rank 1 Lie groups. II. Geom. Funct. Anal. 7 (1997), 561-593. Zbl 0884.20025 MR 1466338

[36] Z. Sela, Endomorphisms of hyperbolic groups I: The Hopf property. Topology 38 (1999), 301-321. Zbl 0929.20033 MR 1660337

[37] Z. Sela, Diophantine geometry over groups I: Makanin-Razborov diagrams. Publ. Math. Inst. Hautes Études Sci. 93 (2001), 31-105. Zbl 1018.20034 MR 1863735

[38] A. Selberg, On discontinuous groups in higher-dimensional symmetric spaces. In Contributions to function theory (Internat. Colloq. Function Theory, Bombay, 1960), Tata Institute of Fundamental Research, Bombay 1960, 147-164. Zbl 0201.36603 MR 0130324

[39] D. S. Silver, Nontorus knot groups are hyperHopfian. Bull. London Math. Soc. 28 (1996), 4-6. Zbl 0836.20049 MR 1356819

[40] S. Smale, A note on open maps. Proc. Amer. Math. Soc. 8 (1957), 391-393. Zbl 0082.16401 MR 0086295 
[41] J. Stallings, On fibering certain 3-manifolds. In Topology of 3-manifolds and related topics (Proc. The Univ. of Georgia Institute, 1961), Prentice-Hall, Englewood Cliffs, N.J., 1962, 95-100. MR 0158375

[42] W. P. Thurston, Three-dimensional manifolds, Kleinian groups and hyperbolic geometry. Bull. Amer. Math. Soc. (N.S.) 6 (1982), 357-381. Zbl 0496.57005 MR 648524

[43] J. Väisälä, Discrete open mappings on manifolds. Ann. Acad. Sci. Fenn. Ser. A I 392 (1966), 1-10. Zbl 0144.22202 MR 0200928

[44] F. Waldhausen, On irreducible 3-manifolds which are sufficiently large. Ann. of Math. (2) 87 (1968), 56-88. Zbl 0157.30603 MR 0224099

[45] J. J. Walsh, Light open and open mappings on manifolds. II. Trans. Amer. Math. Soc. 217 (1976), 271-284. Zbl 0321.57011 MR 0394674

[46] S. C. Wang and Y. Q. Wu, Covering invariants and cohopficity of 3-manifold groups. Proc. London Math. Soc. (3) 68 (1994), 203-224. Zbl 0841.57028 MR 1243842

[47] D. Wilson, Open mappings on manifolds and a counterexample to the Whyburn conjecture. Duke Math. J. 40 (1973), 705-716. Zbl 0273.54008 MR 0320989

[48] D. Witte Morris, An introduction to arithmetic groups. Preliminary version available at http://people.uleth.ca/ dave.morris/

[49] F. Yu and S. Wang, Covering degrees are determined by graph manifolds involved. Comment. Math. Helv. 74 (1999), 238-247. Zbl 0930.57011 MR 1691948

Received September 9, 2009; revised July 27, 2010

M. R. Bridson, Mathematical Institute, University of Oxford, 24-29 St Giles', Oxford OX1 3LB, UK

E-mail: bridson@maths.ox.ac.uk

D. Groves, Department of Mathematics, Statistics, and Computer Science, University of Illinois at Chicago, 322 Science and Engineering Offices (M/C 249), 851 S. Morgan St., Chicago, IL 60607-7045, U.S.A.

E-mail: groves@math.uic.edu

J. A. Hillman, School of Mathematics and Statistics F07, University of Sydney NSW 2006, Australia

E-mail: jonathan.hillman@sydney.edu.au

G. J. Martin, Institute of Information and Mathematical Sciences, Massey University, Albany Campus, Private Bag 102-904, North Shore Mail Centre, Auckland, New Zealand E-mail: g.j.martin@massey.ac.nz 